\title{
Continuous and intermitted nerve monitoring in thyroid surgery: two complementary devices
}

\author{
Hui Sun ${ }^{1}$, Guido Nicola Zanghì ${ }^{2}$, Francesco Freni ${ }^{3}$, Gianlorenzo Dionigi ${ }^{4}$ \\ ${ }^{1}$ Division of Thyroid Surgery, Jilin Provincial Key Laboratory of Surgical Translational Medicine, China-Japan Union Hospital of Jilin University, \\ Changchun 130033, China; ${ }^{2}$ Department of Surgery, Policlinico Vittorio Emanuele University Hospital - General Surgery and Oncology Unit, \\ University of Catania, Catania, Sicily, Italy; ${ }^{3}$ Division ENT Surgery, ${ }^{4}$ Division for Endocrine and Minimally Invasive Surgery, Department of Human \\ Pathology in Adulthood and Childhood "G. Barresi”, University Hospital G. Martino, University of Messina, Messina, Italy \\ Correspondence to: Prof. Gianlorenzo Dionigi. Division for Endocrine and Minimally Invasive Surgery, Department of Human Pathology in \\ Adulthood and Childhood “G. Barresi”, University Hospital G. Martino, University of Messina, Via C. Valeria 1, 98125 Messina, Italy. \\ Email: gdionigi@unime.it.
}

Submitted Jan 20, 2018. Accepted for publication Feb 07, 2018.

doi: $10.21037 /$ gs.2018.02.02

View this article at: http://dx.doi.org/10.21037/gs.2018.02.02

Intraoperative neural monitoring (IONM) is a constitutive procedure in the management of the recurrent laryngeal nerve (RLN) in thyroid surgery. Progress in IONM currently includes devices that intermittently (I-IONM) evaluate RLN functionality and accessories that continuously assess the entire RLN route by permanent vagal nerve stimulation, i.e., continuous intraoperative nerve monitoring (C-IONM) (1). I-IONM is achievable through a monopolar or bipolar hand probe stimulator. C-IONM is a stimulator leaning on the vagus nerve. Both I-IONM and C-IONM wires are connected and interface with the same monitoring software and video system (1). These technologies developed for IONM provide additional and complementary insights into monitored thyroid surgery (Table 1).

C-IONM was subsequently established to overcome the limits of I-IONM. The RLN is still at risk for damage with the use of solely I-IONM proximally to site of intermitted stimulation and between two RLN/vagal stimulations. With I-IONM, the functional integrity of RLN is limited to the short time interval \& site of direct stimulation. I-IONM is able to test the integrity of RLN following the dissection. The surgeon cannot dissect and stimulate at the same time. I-IONM cannot alert the surgeon to prevent injury.
I-IONM records a loss of signal (LOS) after the damage has been done (1).

Thus it can be hypnotize that the only benefit of I-IONM is on the first side of surgical dissection in preventing bilateral paralysis, with stage thyroidectomy (2).

Aim of C-IONM is to recognize imminent RLN stress/ risk of injury, thus avoiding definitive nerve palsy. To overcome the main obstacle of I-IONM: diagnosis of LOS when nerve lesion already has occurred. C-IONM has the possibility to reduce the RLN paralysis, especially the most severe ones (3).

Schneider R et al. study demonstrated that the use of $\mathrm{C}-\mathrm{IONM}$ resulted in significant less severe, definitive permanent RLN palsies compared with only I-IONM use (1). Nevertheless, these two modes of IONM application (I-IONM and C-IONM) form a useful network for the surgeon (Table 1). The two modalities have unique advantages and disadvantages and may be considered complementary devices. Surgeon can not use only and separately C-IONM and exclude the use of I-IONM (Table 1). Either modality may be used as support for surgeon RLN dissection, and the choice of I-IONM device timing is influenced by the surgical step, procedure, difficulties, anatomy, superior laryngeal nerve monitoring 
Table 1 C-IONM and I-IONM are complementary technologies

\begin{tabular}{lcc}
\hline Proprieties & C-IONM & I-IONM \\
\hline Prevent transient RLNP & - & - \\
Prevent permanent RLNP & ++ & - \\
Synchronization of surgical maneuvers & +++ & - \\
Awareness of nerve stress & +++ & + \\
Appreciation of nerve function recovery & +++ & + \\
Mechanism of nerve injury & ++ & + \\
RLN identification, mapping & + & ++ \\
Site of nerve injury & + & ++ \\
Nerve variability, branching & + & ++ \\
Nerve dissection & - & ++ \\
EBSLN monitoring & - & ++ \\
EMG tube malpositioning & ++ & - \\
Documentation & +++ & + \\
\hline
\end{tabular}

EBSLN, external branch superior laryngeal nerve; EMG, electromyography; RLNP, recurrent laryngeal nerve palsy; RLN, recurrent laryngeal nerve. -, not provided; +, dispensed; ++, implemented; +++, completely provided.

(Table 1). A careful evaluation of the costs by using both probes will have to be followed in the near future (4).

Cite this article as: Sun H, Zanghì GN, Freni F, Dionigi G. Continuous and intermitted nerve monitoring in thyroid surgery: two complementary devices. Gland Surg 2018;7(Suppl 1):S80-S81. doi: $10.21037 /$ gs.2018.02.02

\section{Acknowledgements}

None.

\section{Footnote}

Conflicts of Interest: The authors have no conflicts of interest to declare.

\section{References}

1. Schneider R, Sekulla C, Machens A, et al. Postoperative vocal fold palsy in patients undergoing thyroid surgery with continuous or intermittent nerve monitoring. Br J Surg 2015;102:1380-7.

2. Marin Arteaga A, Peloni G, Leuchter I, et al. Modification of the Surgical Strategy for the Dissection of the Recurrent Laryngeal Nerve Using Continuous Intraoperative Nerve Monitoring. World J Surg 2018;42:444-50.

3. Mirallié É, Caillard C, Pattou F, et al. Does intraoperative neuromonitoring of recurrent nerves have an impact on the postoperative palsy rate? Results of a prospective multicenter study. Surgery 2018;163:124-9.

4. Wang T, Kim HY, Wu CW, et al. Analyzing cost-effectiveness of neural-monitoring in recurrent laryngeal nerve recovery course in thyroid surgery. Int J Surg 2017;48:180-8. 